\title{
Estimating the location of a tunnel using correlation and inversion of Rayleigh wave scattering
}

\author{
A. Kaslilar, ${ }^{1}$ U. Harmankaya, ${ }^{1}$ K. Wapenaar, ${ }^{2}$ and D. Draganov ${ }^{2}$ \\ Received 24 October 2013; revised 23 November 2013; accepted 27 November 2013; published 10 December 2013.
}

[1] The investigation of near-surface scatterers, such as cavities, tunnels, abandoned mine shafts, and buried objects, is important to mitigate geohazards and environmental hazards. By inversion of travel times of cross-correlated scattered waves, due to the incident Rayleigh waves, we estimate the location of a near-surface tunnel from seismic field data. The cross correlation eliminates the travel path between a source and a scatterer, thus eliminating the need to know the position of the source, making the estimation of the scatterers' locations dependent only on properties between the receivers and the scatterer. First time using a numerically verified method on seismic field data, we show the potential of the method for estimating the location of a buried scatterer. Citation: Kaslilar, A., U. Harmankaya, K. Wapenaar, and D. Draganov (2013), Estimating the location of a tunnel using correlation and inversion of Rayleigh wave scattering, Geophys. Res. Lett., 40, 6084-6088, doi:10.1002/2013GL058462.

\section{Introduction}

[2] The Earth's subsurface contains heterogeneities at different scales: karstic structures, tunnels, and mine shafts at the engineering scale and sedimentary basins, mountain roots, and mantle plumes at the exploration and global scale. When seismic waves encounter an inhomogeneity comparable with the dominant wavelength's size, they scatter [Sato and Fehler, 1998]. These scattered waves contain information about the medium and can be used for the characterization of scatterers. Surface wave scattering can be effectively used for locating and characterizing near-surface structures in engineering [e.g., Grandjean and Leparoux, 2004; Gelis et al., 2005; Campman and Riyanti, 2007; Kaslilar, 2007; Xia et al., 2007; Chai et al., 2012; Harmankaya et al., 2013] and in exploration and global seismology [e.g., Snieder and Nolet, 1987; Ritzwoller et al., 2002; Rickers et al., 2012].

[3] One of the methods that can be used for determining the scatterers was proposed using arguments from seismic interferometry (SI). SI traditionally retrieves new seismic responses (Green's functions) by cross-correlating wavefields recorded at two locations from many surrounding sources

\footnotetext{
${ }^{1}$ Faculty of Mines, Department of Geophysical Engineering, Istanbul Technical University, Istanbul, Turkey.

${ }^{2}$ Section of Applied Geophysics and Petrophysics, Department of Geoscience and Engineering, Delft University of Technology, Delft, Netherlands.

Corresponding author: A. Kaslilar, Faculty of Mines, Department of Geophysical Engineering, Istanbul Technical University, Istanbul 34469, Turkey. (kaslilar@itu.edu.tr)

(C)2013. American Geophysical Union. All Rights Reserved. 0094-8276/13/10.1002/2013GL058462
}

[Barmin et al., 2011; Campillo and Paul, 2003; Schuster et al., 2004; Shapiro et al., 2005; Snieder, 2004; Wapenaar, 2004]. When the sources do not effectively surround the receivers, next to the physical arrivals also ghost arrivals (also called nonphysical or spurious arrivals) might be retrieved. An example of such a ghost arrival is the correlation of scattered waves at two receivers: Snieder et al. [2008, equation (5), term T4]. This term is a result of the optical theorem and is formally canceled by other terms in the correlation. Here we use this scattered energy by performing an isolated correlation. This is in the spirit of other efforts to use ghost signals in the correlation in SI [Mikesell et al., 2009; Bharadwaj et al., 2012; Halliday and Curtis, 2009; Draganov et al., 2012; Meles and Curtis, 2013].

[4] When the objective is to retrieve only a specific part of the Green's function, for example, scattered waves between receivers, then only some of the surrounding sources may be sufficient. Those sources should be located in the stationary phase region [Snieder, 2004], i.e., around the specular ray that passes from a source to one of the receivers (direct field), scatters and is recorded at the other receivers (scattered field). When only one source is present and only scattered fields are correlated, only ghost arrivals will be retrieved [Harmankaya et al., 2013]. We use such correlations of scattered arrivals to estimate the location of a scatterer. Here the scattered waves are body waves, caused by the incident Rayleigh waves. We apply the method for the first time to seismic field data and successfully estimate the location of a buried tunnel.

\section{Method}

[5] We utilize a method inspired by SI, but we make use of only one source and an array of receivers. We take the recordings at the receivers from the source and isolate only the scattered wavefield [Harmankaya et al., 2013] by muting all other arrivals. We select a reference receiver, at the virtual source (VS) position, on the isolated record, and cross correlate all the traces with the trace at the virtual source position. This eliminates the common travel path from the real source to the scatterer and reveals the difference in the travel time the scattered wavefield will have when traveling only between the scatterer and the VS and between the scatterer and the rest of the receivers. This results in the retrieval of ghost-scattered waves. The location of the scatterer $(x, y$, and $z$ ) is estimated by inverting for the following ghost travel time relation:

$$
\begin{aligned}
t_{i}= & \frac{1}{V}\left\{\left[\left(x^{i}-x\right)^{2}+\left(y^{i}-y\right)^{2}+\left(z^{i}-z\right)^{2}\right]^{1 / 2}\right. \\
& \left.-\left[\left(x^{\mathrm{VS}}-x\right)^{2}+\left(y^{\mathrm{VS}}-y\right)^{2}+\left(z^{\mathrm{VS}}-z\right)^{2}\right]^{1 / 2}\right\},
\end{aligned}
$$

where $V$ is the wave velocity and $i$ is the receiver index. Retrieval of the ghost field for one virtual source is sufficient 


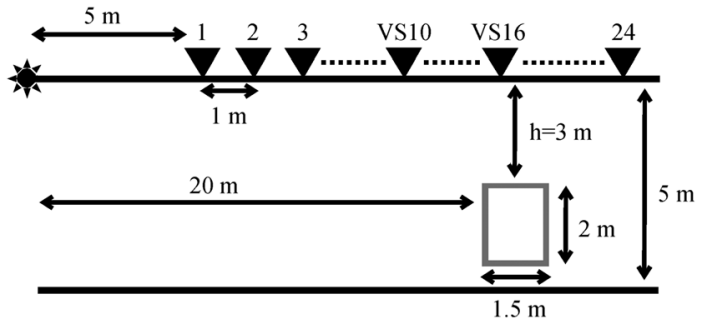

Figure 1. Acquisition geometry of the seismic field data above the tunnel (not to scale). The source (star), receivers (triangles), and the tunnel with masonry (rectangle). VS10 and VS16 are selected virtual source locations used in the location estimation. The source location is set as the origin of the acquisition geometry.

for locating the scatterer; however, more virtual sources can be used for comparison of the estimations and averaging.

[6] Even though the method was explained using arguments from SI, it has another explanation as well. If two receivers record the source response along the same raypath in a smooth medium, then the correlation of the recordings would eliminate the time for propagation from the source to the first receiver and would show the travel time difference for the wave to travel between the two receivers. Let us now add a scatterer in the medium. The correlation will show the travel time difference between the direct arrivals and the scattered arrivals. If the direct arrivals are eliminated from the recordings, then the correlation of the scattered arrivals would eliminate the common travel time for the wave to propagate from the source to the scatterer and the result would show the travel time difference from the wave to propagate from the scatterer to each of the receivers. This method has parallels with earthquake location algorithms, where the travel time difference between two direct arrivals, namely of $S$ and $P$ waves to a seismic station provides a relative distance to the earthquake [Stein and Wysession, 2006].
[7] The considered problem is weakly nonlinear in the unknown positions, so we linearize it to write it in a matrix-vector form as $\Delta \mathbf{d}=\mathbf{G} \Delta \mathbf{m}$ and solve it iteratively. The vector $\Delta \mathbf{d}=\mathbf{t}^{\text {obs }}-\mathbf{t}^{\text {calc }}$ is the difference between an observed ghost arrival time, $t^{\text {obs }}$, and a calculated one, $t^{\text {calc }}$, on the basis of an assumed scatterer position (equation (1)). The unknown coordinates of the scatterer are denoted by the vector $\Delta \mathbf{m}$, while the Jacobian (sensitivity) matrix is represented by $\mathrm{G}$. We solve the inverse problem using damped singular value decomposition and calculate the uncertainties of the estimations by the model covariance matrix using a coverage factor 2 , which provides a confidence level of $95 \%$. In this example, the wave velocity between the receivers and a scatterer is considered as known and it is estimated from the arrivals in the record. Velocity can also be included as an unknown parameter in $\Delta \mathbf{m}$, to be estimated along with the scatterer location. Due to the nonlinear nature of the problem, as long as estimating the velocity from the data is possible, we prefer to use it as known parameter to increase the accuracy of the locations.

\section{Estimation of the Location of a Tunnel Acting as Scatterer}

[8] We apply the method proposed by Harmankaya et al. [2013] for the first time to seismic field data collected above an a priori known cavity by BRGM (Bureau de Recherche Géologique et Minière) at Jargeau site, Loiret, France [Leparoux et al., 2000]. The data are recorded along a $63 \mathrm{~m}$ long profile with many shots and 24 channel receivers. The temporal sampling, nearest offset, shot, and receiver group intervals are $1 \mathrm{~ms}, 5 \mathrm{~m}, 1 \mathrm{~m}$, and $1 \mathrm{~m}$. respectively. The cavity is a tunnel with masonry, at $3 \mathrm{~m}$ depth from the surface. The size of the tunnel is $2 \mathrm{~m}$ by $1.5 \mathrm{~m}$ in height and width, respectively (Figure 1). The dominant frequency of the records is about $40 \mathrm{~Hz}$. We use the record in Figure $2 \mathrm{a}$ and calculate the direct Rayleigh wave velocity from the slope $\left(V_{\mathrm{R}}=\Delta x / \Delta t\right)$ of the direct Rayleigh wave arrival as $180 \mathrm{~m} / \mathrm{s}$. Considering
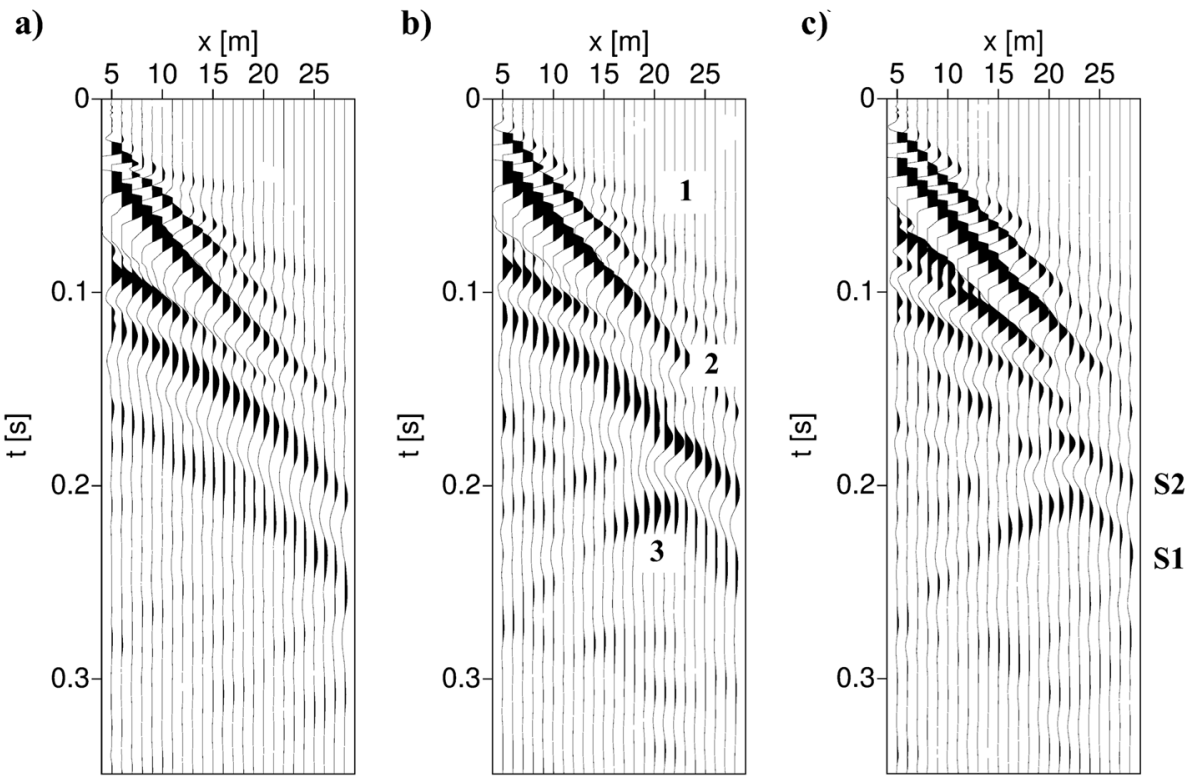

Figure 2. Common shot records (a) for shot 10, assumed incident wavefield, and (b) for shot 19, assumed total wavefield. The direct and refracted body waves (1), direct Rayleigh waves (2), and scattered waves (3) are indicated. (c) The scattered wave field obtained by taking the difference of Figures $2 \mathrm{a}$ and $2 \mathrm{~b}$. S1 and S2 represent the diffractions from the right and left corners of the tunnel, respectively. 

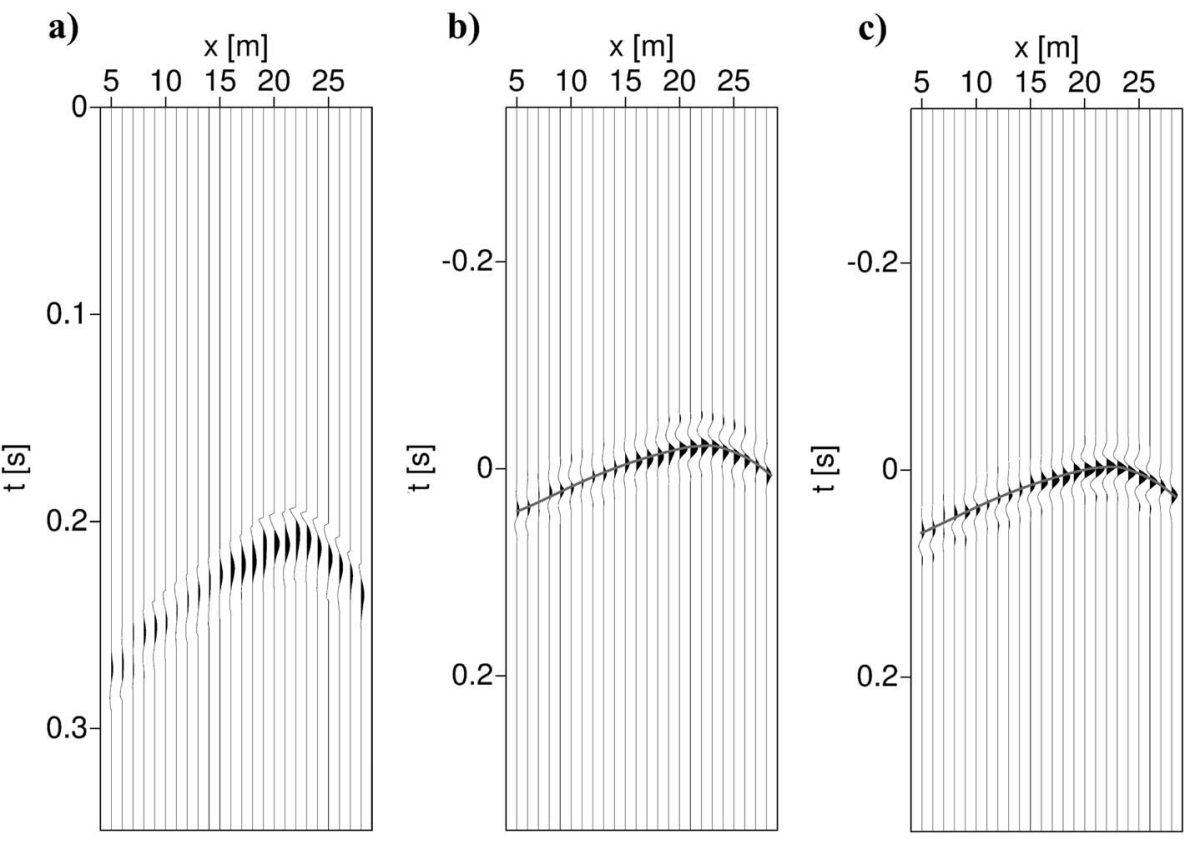

Figure 3. (a) Isolated scattered wavefield S1. Ghost-scattered arrivals retrieved by seismic interferometry applied to Figure $3 \mathrm{a}$ for the virtual sources at (b) VS10 and (c) VS16. The gray lines show the picked travel times.

the dominant frequency and the velocity, we calculate the dominant Rayleigh wavelength as $4.5 \mathrm{~m}$, which is comparable to the size and the depth of the scatterer. As the tunnel is buried, after the scattering of the incident Rayleigh wave field, we obtain body waves. Therefore, for estimating the location of the scatterer, we use $S$ wave velocity $\left(V_{S}\right)$ as $200 \mathrm{~m} / \mathrm{s}$, calculated by the relation $V_{\mathrm{R}} \cong 0.9 V_{S}$.

[9] To estimate the $x$ and $z$ coordinate of the tunnel, first we need an isolated scattered wave field. In this case, we make advantage of having several shot gathers. Each shot gather consists of 24 receivers and a source at $5 \mathrm{~m}$ to the left of the leftmost receiver. We chose two of them: one above and the other away to the left (not above) of the tunnel. The shot gather away from the tunnel is considered as the incident wavefield in the absence of the scatterer (Figure 2a), and the shot gather above the tunnel is considered as the total wavefield including the scattered wavefield (Figure 2b). The difference of the assumed total and incident wavefields (Figures $2 \mathrm{~b}$ and $2 \mathrm{a}$ ) results as the scattered wavefield (Figure 2c) due to the tunnel, provided that the medium properties are not changing significantly in the lateral direction. In Figure $2 c$ the scattered wavefield due to the incidence of Rayleigh waves can be clearly observed. As a first location estimation from the scattered wavefield (Figure 2c) we chose the second hyperbola with the least distorted waveforms (marked as S1 in Figure 2c), which is due to the right corner of the tunnel. To remove the remnants of the direct arrivals (i.e., incident wavefield) and also the other redundant arrivals, we mute them out, to obtain a clean scattered wave hyperbola (Figure 3a). In cases where only one shot gather is available, a frequency-wavenumber (f-k) filter [Buttkus, 2000] can be applied to the data to isolate the scattered wavefield. By f-k filtering, the direct wavefield (linear arrivals) can be separated according to its dip, and the scattered wavefield can be obtained by substracting this direct wavefield from the total wavefield [Harmankaya et al., 2013, section 3, for example].
[10] We then choose VS locations for which we want to retrieve the ghost-scattered waves needed for the inversion. For the shot gather above the tunnel, we set the source position as the origin of the coordinate system and choose the receivers 10 and 16 as the VS locations, i.e., VS10 (14 m) and VS16 $(20 \mathrm{~m})$ in Figure 1. The VS locations are arbitrarily chosen, however, it should be noted that a trace with a waveform that is representative of the waveforms of other traces, will give higher correlation and better arrival time readings. For each VS location, the trace at that receiver is cross-correlated with itself and all other traces on the isolated scattered wavefield. In this way the ghost-scattered waves are retrieved (Figures $3 \mathrm{~b}$ and $3 \mathrm{c}$ ). The correlation of the VS with itself results in an arrival at time $t=0 \mathrm{~s}$, whereas the arrivals in the other traces are shifted in agreement with equation (1).

[11] To perform the inversion, one has to pick the ghost travel times by considering the maximum amplitude of the retrieved ghost arrivals. When the scatterer is different from a point scatterer, the wavefield at the scatterer may undergo scattering, reflection, and refraction and exhibit directionality. In this case, the scattered branches will have different slopes and the point scatterer model (equation (1)) may not work accurately. As the boundary of the scatterer generates scattered wavefields, the travel times at the apex and at one side of the scattering hyperbola can be used for picking the travel times, and for the opposite branch, the symmetry of the readings can be accepted. In the field data, we considered the left and the right tunnel corners to be each a point scatterer and picked the complete ghost travel times for inversion. The updates of the model parameters after each iteration are given in Figure 4b. The inversion is stopped when the changes in the model parameters become less than $0.1 \%$. The estimated coordinates of the tunnel are listed in Table 1 for VS10 and VS16, with their actual locations. We also apply the same procedures to $\mathrm{S} 2$ in Figure 2c, which is the scattering from the left corner of the tunnel. The left branch of the scattered wavefield of S2 has distorted 
a)

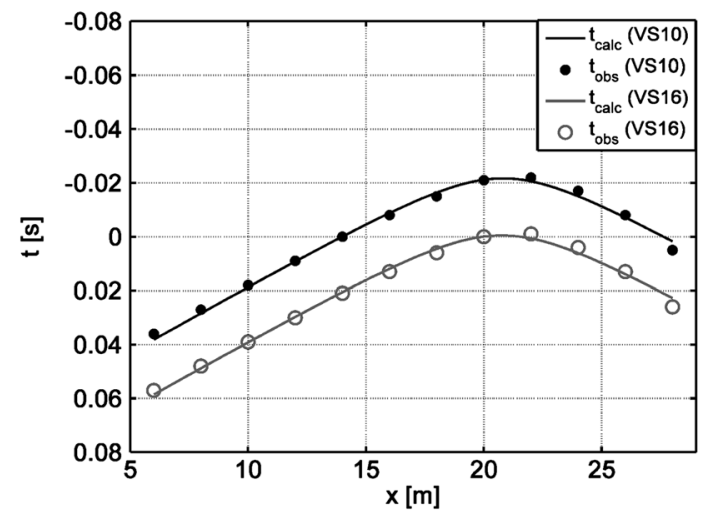

b)
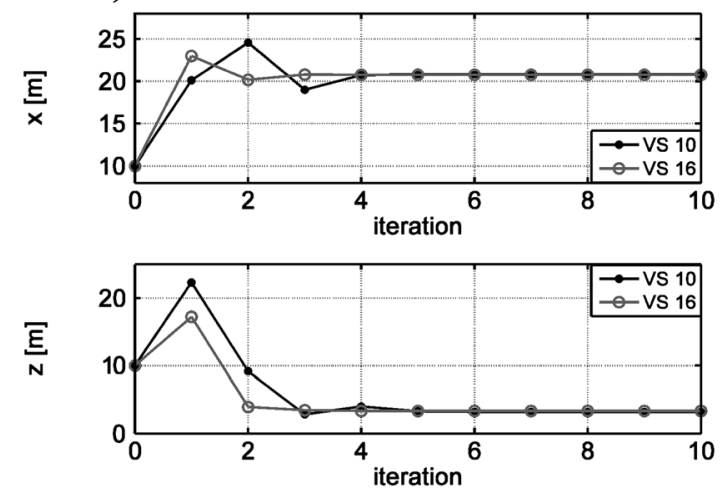

Figure 4. (a) Observed (dots) and calculated (solid line) travel times; (b) Estimated locations ( $x$ and $z$ ) of the tunnel's right corner for the virtual sources 10 and 16 . The values at the zeroth iteration correspond to the initial parameters for the inversion.

waveforms; therefore, we select the VS trace from the right side of S2, VS20, and estimate the location of the tunnel again (S2 in Table 1).

[12] We calculate the errors in the estimated model parameters by

$$
E_{m}=\left|\frac{m_{\mathrm{act}}-m_{\mathrm{est}}}{m_{\mathrm{act}}}\right| \times 100,
$$

where $m_{\text {act }}$ and $m_{\text {est }}$ are the actual and estimated model parameters $(x$ or $z)$, respectively. We show that we estimate the location of the tunnel with less than $11 \%$ error.

[13] For the estimated model parameters at the final iteration, we calculate the ghost travel times and plot them together with the picked (observed) ones in Figure 4a for VS10 and VS16. To quantify the visually observed agreement, we use the following relation

$$
E_{t}=\frac{\sum_{k=1}^{N}\left(t_{k}^{\mathrm{obs}}-t_{k}^{\mathrm{calc}}\right)^{2}}{\sum_{k=1}^{N}\left(t_{k}^{\mathrm{calc}}\right)^{2}} \times 100
$$

and show the results in Table 1. It is seen than there is less than $1 \%$ error between the observed and the calculated travel times.

\section{Discussion and Conclusion}

[14] We showed results from a method inspired by seismic interferometry and inversion to locate scatterers. Only one source is sufficient to locate the scatterers, provided that the scattered wave field is well isolated from the total wave field. We showed the potential of the method on seismic field data at geotechnical scale and successfully estimated the location of a buried tunnel.

[15] In the field data example, we used single-scattered events from the corners of the tunnel in a laterally homogeneous medium. Structures like karst cavities, tunnels, and mine shafts at the engineering scale and sedimentary basins and mountain roots in exploration and global scale can act as single scatterers. As in our example, many shot gathers are common in geotechnical- and exploration-scale experiments, and the scattered wavefield can be isolated by extracting two wavefields as explained in section 3, if background medium properties can be considered alike. In case of a single shot gather and a single scatterer inline with the receiver line, the isolation of the scattered wavefield can be provided by frequency-wavenumber filtering. When a single or many scatterers are present off to the side of the receiver line, to locate the scatterers, a common shot with two parallel or orthogonal receiver lines are required. The selection and isolation of the scattered wavefields in such geometries are relatively easy if small numbers of scatterers are available in the medium. In the presence of many scatterers, the selection of the hyperbola that belongs to the same scatterer may be difficult. For parallel receiver lines using the apices observed around the same receiver locations could overcome these difficulties; for orthogonal lines, selection of the correct hyperbola becomes challenging. One practical solution is to perform the inversion. If hyperbolas corresponding to different scatterers are selected, the observed and the calculated travel time curves will not match and another potential scattering hyperbola can be considered for location purposes.

\begin{tabular}{|c|c|c|c|c|c|c|}
\hline & VS \# & $\mathrm{AL}(\mathrm{m}) x / z$ & $x \pm \sigma_{x}(\mathrm{~m})$ & $z \pm \sigma_{y}(\mathrm{~m})$ & $E_{t}(\%)$ & $E_{m}(\%) x / z$ \\
\hline \multirow[t]{2}{*}{ Average } & $\begin{array}{l}10 \\
16\end{array}$ & $21.5 / 3.0$ & $\begin{array}{c}S 1 \\
20.80 \pm 0.11 \\
20.76 \pm 0.13 \\
20.78 \pm 0.12\end{array}$ & $\begin{array}{l}3.18 \pm 0.39 \\
3.34 \pm 0.19 \\
3.26 \pm 0.31\end{array}$ & $\begin{array}{l}0.64 \\
0.26\end{array}$ & $\begin{array}{c}3.2 / 6.0 \\
3.4 / 11.3 \\
3.3 / 8.6\end{array}$ \\
\hline & 20 & $20.0 / 3.0$ & $\begin{array}{c}S 2 \\
19.98 \pm 0.08\end{array}$ & $3.15 \pm 0.18$ & 0.16 & $0.1 / 5.0$ \\
\hline
\end{tabular}

Table 1. The Estimated Model Parameters for Virtual Source (VS \#) Locations and for the Geometry Given in Figure $1^{\mathrm{a}}$

\footnotetext{
${ }^{\mathrm{a}}$ The actual location of the tunnel (AL), the estimated parameters $\left(x\right.$ and $z$ ) with their $95 \%$ confidence levels $(1.96 \sigma)$, percentage errors on the travel times $\left(E_{t}\right)$, and model parameters $\left(E_{m}\right)$ are also given. S1 and S2 indicate that the estimated horizontal coordinate is for the right and left corners of the tunnel, respectively.
} 
[16] The detection, monitoring, and stabilization of nearsurface structures are important to mitigate geohazards and environmental hazards, and our method can be considered as one of the alternatives for detecting and locating scatterers. The method is not restricted to the Rayleigh wave scattering discussed in this paper. The same methodology holds for other types of scattering experiments. An important advantage of the presented technique is that the method is independent of the wave propagation from the source to the scatterer. As a result, we foresee application of the method in wavescattering problems not only at geotechnical scale but also at all length scales.

[17] Acknowledgments. We are grateful to Gilles Grandjean and Arnand Bitri from the BRGM France for allowing us to use the data. We thank Kasper van Wijk for his stimulating discussions and the Colorado School of Mines for providing the Seismic Un*x [Cohen and Stockwell, 2012] package as open source software. We thank the Editor, Anatoli L. Levshin, and an anonymous reviewer for their valuable comments. This work is supported by TUBITAK with the project CAYDAG-110Y250. A.K. and U.H. gratefully acknowledge this financial support. The investigations of D.D. are supported by the Division for Earth and Life Sciences (ALW) with financial aid from the Netherlands Organization for Scientific Research (NWO).

[18] The Editor thanks Anatoli Levshin and an anonymous reviewer for their assistance in evaluating this paper.

\section{References}

Barmin, M. P., A. L. Levshin, Y. Yang, and M. H. Ritzwoller (2011), Epicentral location based on Rayleigh wave Empirical Green's Functions from ambient seismic noise, Geophys. J. Int., 184, 869-884, doi:10.1111/ j.1365-246X.2010.04879.x.

Bharadwaj, P., G. Schuster, W. Dai, and I. Mallinson (2012), Theory of super-virtual refraction interferometry, Geophys. J. Int., 188(1), 263-273, doi:10.1111/j.1365-246X.2011.05253.x.

Buttkus, B. (2000), Spectral Analysis and Filter Theory in Applied Geophysics, Springer-Verlag, Berlin, Heidelberg, New York.

Campillo, M., and A. Paul (2003), Long-range correlations in the diffuse seismic coda, Science, 299, 547-549, doi:10.1126/science.1078551.

Campman, X., and C. D. Riyanti (2007), Non-linear inversion of scattered seismic surface waves, Geophys. J. Int., 171, 1118-1125, doi:10.1111/ j.1365-246X.2007.03557.x.

Chai, H. Y., K. K. Phoon, S. H. Goh, and C. F. Wei (2012) Some theoretical and numerical observations on scattering of Rayleigh waves in media containing shallow rectangular cavities, J. Appl. Geophys., 83, 107-119, doi:10.1016/j.jappgeo.2012.05.005. ISSN:0926-9851.

Cohen, J. K., and J. W. Stockwell Jr. (2012), CWP/SU: Seismic Un*x Release No. 43: An open source software package for seismic research and processing, Center for Wave Phenomena, Colorado School of Mines.

Draganov, D., K. Heller, and R. Ghose (2012), Monitoring $\mathrm{CO}_{2}$ storage using ghost reflections retrieved from seismic interferometry, Int. J. Greenh. Gas Control, 11S, S35-S46, doi:10.1016/j.ijggc.2012.07.026.
Gelis, C., D. Leparoux, J. Virieux, A. Bitri, S. Operto, and G. Grandjean (2005), Numerical modeling of surface waves over shallow cavities, J. Environ. Eng. Geophys., 10(2), 111-121, doi:10.2113/JEEG10.2.111.

Grandjean, G., and D. Leparoux (2004), The potential of seismic methods for detecting cavities and buried objects: Experimentation at a test site, J. Appl. Geophys., 56(2), 93-106, doi:10.1016/j.jappgeo.2004.04.004.

Halliday, D. F., and A. Curtis (2009), Seismic interferometry of scattered surface waves in attenuative media, Geophys. J. Int., 178, 419-446, doi:10.1111/j.1365-246X.2009.04153.x.

Harmankaya, U., A. Kaslilar, J. Thorbecke, K. Wapenaar, and D. Draganov (2013), Locating near-surface scatterers using non-physical scattered waves resulting from seismic interferometry, J. Appl. Geophys., 91, 66-81, doi:10.1016/j.jappgeo.2013.02.004.

Kaslilar, A. (2007), Inverse scattering of surface waves: Imaging of near-surface heterogeneities, Geophys. J. Int., 171, 352-367, doi:10.1111/j.1365246X.2007.03524.x.

Leparoux, D., A. Bitri, and G. Grandjean (2000), Underground cavity detection: A new method based on seismic Rayleigh waves, EJEEG, 5 , 33-53.

Meles, A. G., and A. Curtis (2013), Physical and non-physical energy in scattered wave source-receiver interferometry, J. Acoust. Soc. Am., 133(6), 3790-3801, doi:10.1121/1.4802825.

Mikesell, T. D., K. van Wijk, A. Calvert, and M. Haney (2009), The virtual refraction: Useful spurious energy in seismic interferometry, Geophysics, 74(3), A13-A17, doi:10.1190/1.3095659.

Rickers, F., A. Fichtner, and J. Trampert (2012), Imaging mantle plumes with instantaneous phase measurements of diffracted waves, Geophys. J. Int., 190, 650-664, doi:10.1111/j.1365-246X.2012.05515.x.

Ritzwoller, M., N. Shapiro, M. P. Barmin, and A. L. Levshin (2002), Global surface wave diffraction tomography, J. Geophys. Res., 107(B12), 2335, doi:10.1029/2002JB001777.

Sato, H., and M. Fehler (1998), Seismic Wave Propagation and Scattering in the Heterogeneous Earth, AIP Press, New York.

Schuster, G. T., J. Yu, J. Sheng, and J. Rickett (2004), Interferometric/ daylight imaging, Geophys. J. Int., 157, 838-852, doi:10.1111/j.1365246X.2004.02251.x.

Shapiro, N. M., M. Campillo, L. Stehly, and M. H. Ritzwoller (2005), High resolution surface-wave tomography from ambient seismic noise, Science, 307, 1615-1618, doi:10.1126/science.1108339.

Snieder, R. (2004), Extracting the Green's function from the correlation of coda waves: A derivation based on stationary phase, Phys. Rev. E, 69, 046610, doi:10.1103/PhysRevE.69.046610.

Snieder, R., and G. Nolet (1987), Linearized scattering of surface waves on a spherical Earth, J. Geophys., 61, 55-63.

Snieder, R., K. van Wijk, M. Haney, and R. Calvert (2008), Cancellation of spurious arrivals in Green's function extraction and the generalized optical theorem, Phys. Rev. E, 78, 036606, doi:10.1103/PhysRevE.78.036606.

Stein, S., and M. Wysession (2006), An Introduction to Seismology, Earthquakes, and Earth Structure, Wiley-Blackwell, Hoboken, N. J.

Wapenaar, K. (2004), Retrieving the elastodynamic Green's function of an arbitrary inhomogeneous medium by cross correlation, Phys. Rev. Lett., 93(25), 254301, doi:10.1103/PhysRevLett.93.254301.

Xia, J., J. E. Nyquist, Y. X. Xu, M. J. S. Roth, and R. D. Miller (2007), Feasibility of detecting near-surface feature with Rayleighwave diffraction, J. Appl. Geophys., 62(3), 244-253, doi:10.1016/j. jappgeo.2006.12.002. 